Conclusion The $\mathrm{R}^{4} \mathrm{Q}$ family of catheters incorporates novel technology for CA. This series demonstrates a high FPE and similar rates of SR compared with published data using other aspiration catheters. Further prospective studies of this technology are planned to evaluate its clinical efficacy.

Disclosures R. Torabi: None. M. Mokin: 1; C; NIH R21NS109575. 2; C; Medtronic, Canon Medical, Cerenovus. 4; C; Serenity Medical, Synchron, Endostream, VICIS. Z. Ren: None. A. Siddiqui: 1; C; NIH/NINDS, Cerenovus, Medtronic, Microvention, MUSC, Penumbra. 2; C; Amnis Therapeutics, Boston Scientific, Canon Medical Systems USA, Cerebrotech Medical Systems, Cerenovus, Corindus, Endostream Medical, Guidepoint Global Consulting, Imperative Care, Integra, Medtronic, Microvention, Minnetronix Neuro, Northwest University, Penumbra, Q’Apel Medical, Rapid Medical, Rebound Therapeutics, Serenity Medical, Silk Road Medical, StimMed, Stryker, Three Rivers Medical, VasSol, W. L. Gore \& Associates. 4; C; Adona Medical, Amnis Therapeutics, BlinkTBI, Buffalo Technology Partners, Cardinal Consultants, Cerebrotech Medical Systems, Cognition Medical, Endostream Medical, Imperative Care, International Medical Distribution Partners, Neurovascular Diagnostics, Q'Apel Medical, Rebound Therapeutics, Rist Neurovascular, Sense Diagnostics, Serenity Medical, Silk Road Medical, Spinnaker Medical, StimMed Synchron, Three Rivers Medical, VasSol, W.L Gore \& Associates. E. Levy: 2; C; Claret Medical, GLG Consulting, Guidepoint Global, Imperative Care, Medtronic, Rebound Therapeutics, StimMed. 4; C; NeXtGen Biologics, RAPID Medical, Claret Medical, Cognition Medical, Imperative Care, Rebound Therapeutics, StimMed, Three Rivers Medical. 6; C; National PI: Medtronic, Steering committees for SWIFT Prime and SWIFT Direct trials, Honorarium for Training and Lectures: Medtronic, Advisory Board: Styker (AIS clinical advisory board), NeXtGen Biologics, MEDX, Cognition Medical, Endostream Medical, Site PI: Microvention (Confidence Study). M. Waqas: None. A. Arthur: 1; C; Balt, Cerenovus, Medtronic, Microvention, Penumbra, Siemens, Stryker. 2; C; Johnson \& Johnson, Microvention, Medtronic, Penumbra, Scientia, Siemens, Stryker. 4; C; Bendit, Cerebrotech, Endostream, Magneto, Marblehead, Neurogami, Serenity, Synchron, Triad Medical, Vascular Simulations. D. Hoit: 2; C; Medtronic, Microvention. 4; C; Silver Bullet, Cerebrotech, Marblehead Medical. C. Nickele: 1; C; Microvention. 2; C; Leica. V. Inoa: None. B. Jankowitz: 2; C; Stryker, Medtronic.

\section{E-067 REDUCING TIME TO REVASCULARIZATION IN ACUTE ISCHEMIC STROKE}

H Shownkeen, A Doerr*, S Togbi-Wonyo, B Fill, A Mazumdar, D Pandya, A Lindstrom, M Shownkeen. Northwestern Medicine Central Dupage Hospital, Winfield, IL

\subsection{6/neurintsurg-2020-SNIS.103}

Background Endovascular treatment (EVT) of large vessel occlusions in the treatment of disabling strokes is more effective if reperfusion is achieved quickly. Streamlining protocols from patient presentation to groin puncture can lead to improved timelines to treatment and functional outcomes.

Purpose The purpose of this study was to identify the impact of specific hospital based process improvement strategies in the acute ischemic stroke patient population undergoing endovascular therapy with the specific intent to decrease median arrival to groin puncture. Our multidisciplinary events to review pre and intraprocedure work flow as part of a process improvement (PI) project began in November 2018 during fiscal year (FY) 2019 (9/1/2018-9/1/2019) and implementation of recommendations began FY 2020 (9/1/19. 12/31/19).

Methods The study includes a pre- and post-intervention retrospective review of consecutive patients 18 years or older, hospital admission between September 1, 2016 and December 31, 2019, who underwent EVT for treatment of acute ischemic stroke. Patients experiencing acute stroke within an established hospital admission were excluded from analysis. The primary outcome variables were time from arrival to groin puncture and first pass during the acute ischemic stroke admission. The data points were collected from internal stroke review dataset and anonymized prior to analysis. All but two of our cases used general anesthesia.

Results In FYs 2017 (9/1/16-9/1/17) and 2018 (9/1/17-9/1/ 18) before intervention our median door to needle times were 94 minutes in FY 2017 (37 patients) and 113 minutes in FY 2018 (48 patients). Our median door to first pass times were 114 minutes in FY 2017 and 139 minutes in FY 2018. Post intervention our door to groin puncture times were 64 minutes in FY 2019 (64 patients) and 56 minutes in FY 2020 (35 patients). Our median door to first pass times were 86 minutes in FY 2019 and 74 minutes in FY 2020. The drop in door to groin puncture and door to first pass times pre and post intervention were statistically significant $(\mathrm{P}<0.05)$.

Conclusions Having multidisciplinary team events dedicated to PI alone (which provided education and awareness to multiple stakeholders) resulted in a substantial decrease in median door to groin puncture and door to first pass times. Implementation of hospital based PI initiatives to improve work flow including: early endovascular team activation, a streamlined transport process, a streamlined patient preparation process in the lab, and utilization of a feedback tool resulted in continued improvement in door to groin puncture and first pass times.

Disclosures H. Shownkeen: None. A. Doerr: None. S. TogbiWonyo: None. B. Fill: None. A. Mazumdar: None. D. Pandya: None. A. Lindstrom: None. M. Shownkeen: None.

\section{E-068 IMPROVING EFFICIENCY OF ACUTE ISCHEMIC STROKE THERAPIES: REDUCING DOOR-TO-NEEDLE AND DOOR- TO-PUNCTURE TIME}

V Chin*, K Yeboah, A Balushi, A Guthrie, K Christopher, R Edgell. Saint Louis University, Saint Louis, MO

\subsection{6/neurintsurg-2020-SNIS.104}

Introduction/Purpose Early recanalization has been associated with a higher likelihood of favourable clinical outcome in acute ischemic stroke (AIS). It is imperative that stroke systems of care are set up to minimize workflow latencies that delay initiation of reperfusion therapies, i.e. IV tPA and mechanical thrombectomy (MT). Guidelines recommend a maximum door-to-needle (DTN) time of 60 minutes and a door-to-groin puncture time (DGPT) of 90 minutes in 50\% of cases of large vessel occlusion (LVO). We aimed to evaluate various workflow latencies during stroke codes, including door-to-needle and door-to-groin puncture times, prior to and 